\title{
Implications of a Low-Carbohydrate, High-Fat Diet on Heart Size in a Murine Model
}

\author{
Katelin Gresty $^{1}$, Celena Scheede-Bergdahl ${ }^{2}$, Andreas Bergdahl ${ }^{1, *}$ \\ ${ }^{1}$ Department of Exercise Science, Concordia University, Montreal, H4B 1R6, Qc, Canada \\ ${ }^{2}$ Department of Kinesiology \& Physical Education, McGill University, Montreal, H2W 1S4, Qc, Canada \\ *Corresponding Author: andreas.bergdahl@concordia.ca
}

Copyright $(\subseteq 2014$ Horizon Research Publishing All rights reserved.

\begin{abstract}
Despite growing popularity of carbohydrate restricted diets, consequences of long term use remain unknown. The current study evaluated the effects of low carbohydrate/high fat (LCHF) diets on heart size and cardiac glycogen content in young mice. Young mice (age $21 \mathrm{~d}, \mathrm{n}=$ 24) and mature mice (age $84 \mathrm{~d}, \mathrm{n}=18$ ) were placed on either a LCHF, a western (WD) or a control diet for 12 weeks. Upon completion of the dietary intervention, heart wet weights were measured to compute the weight-to-tibia length ratio. Sections of cardiac tissue were stained with periodic acid and Schiff reagent to visualize glycogen. Mice following the LCHF diet displayed smaller heart weight-to-tibia length ratio when compared to controls. The trend was observed in both young $(\mathrm{p}=0.012)$ and mature $(\mathrm{p}$ $=0.024)$ mice. Cardiac tissue from mice consuming the LCHF diet had a reduced percentage of total area stained positive for glycogen when compared to mice following the WD diet $(\mathrm{p}=0.016)$. This study shows that a LCHF diet reduces heart weight in young and mature mice. Alterations in intercellular cardiac glycogen content may contribute to differences observed in heart weight. LCHF diets should be recommended with caution as long term effects on the heart are unknown.
\end{abstract}

Keywords Heart, Cardiomyocytes, Nutrition, Glycogen stores, Remodeling, Young vs. Old, Mice

\section{Introduction}

The incidence of childhood obesity in North America and around the world has risen significantly over the past decade. Childhood obesity is associated with altered bone development, hyperlipidemia, glucose intolerance, and hepatic steatosis [1]. In more severe, cases children are acquiring hypertension and sleep apnea [1]. In 2010, approximately $6.7 \%$ of all children were considered to be overweight or obese with prevalence expected to rise to $9.1 \%$ in the year 2020 [2]. With such a large percentage of the Western world struggling with childhood obesity, clinicians are constantly searching for healthy and effective weight management strategies. Many of these strategies focus on diets involving caloric restriction or macronutrient manipulation. A low-carbohydrate, high-fat diet (LCHF), popularized by Dr. Robert Atkins in 1972, was the first of many trendy diets where dieters will significantly limit the calories consumed from carbohydrates while simultaneously increasing caloric intake from fat and protein sources [3]. By lowering the carbohydrate intake, glycogen stores become depleted leaving the body to use fats as a primary source of ATP production. In overweight and obese adults, this type of LCHF diet has been associated with accelerated weight loss, decreases in systolic and diastolic blood pressure, improved LDL and HDL cholesterol profiles, and reduced insulin levels [4, 5]. More recently, clinicians have been recommending LCHF diets to children and adolescents in order to achieve with weight loss. Improvements in body mass index Z-scores and lipid profiles in adolescents following a LCHF diet parallel the changes seen in the adult population [6]. However, there are still safety concerns that exist when this diet is used in the adult population that could be even more detrimental to children and adolescents. In a murine model, it has been shown that LCHF diets may lead to an accelerated atherosclerotic process due to increased cholesterol retention in the vascular walls [7]. It has also been shown that LCHF diets impair cardiac phosphate metabolism and cognitive performance [8]. During short-term use, a LCHF diet does not appear to have a negative effect on overall cardiac function, but over an extended period the effects of a prolonged state of ketosis on the heart and the overall growth and development are unknown [8]. It is well accepted that the heart will rely predominantly on fatty acids for energy production; however various stresses can stimulate a greater reliance on glycogen. The importance of glycogen in the ischemic heart and hypertrophied heart are well documented $[9,10]$. It has also been shown that when the workload of the heart is increased, such as after stimulation by epinephrine or with exercise, the cardiomyocytes will depend more heavily on glycogen as an energy source [11-13]. More recent studies have shown that glycogen in the developing embryonic heart is crucial for 
survival, with impairments in glycogen synthase activity being related to congenital heart disease [14].

The present investigation evaluates the effects of a LCHF diet compared to a high-fat western diet (WD) and a control diet on heart size and glycogen content in young hearts. We hypothesize that prolonged use of a LCHF diet negatively impacts the growth and development of the young heart through alterations in cellular arrangement and glycogen content. We expect to see similar changes in both young and mature hearts. Furthermore, we hypothesize that these changes will be more prevalent in the LCHF group than the WD and control groups due to a lack of available carbohydrate.

\section{Materials and Methods}

\subsection{Animals}

C57BL/6 mice were chosen as a suitable murine model for this investigation as they are known to demonstrate similar characteristics to humans following a LCHF diet; including weight loss, improved glucose tolerance, and decreased serum insulin levels [15]. For our young model (YOUNG), 24 male C57BL/6 pups were weaned at 21 days after birth and placed immediately on their assigned diet (refer to Table 1 for diet compositions). In our mature model (MATURE), at 12 weeks of age, 18 male C57BL/6 mice had their standard chow replaced with the assigned diet. All diets were randomly assigned. Only male mice were used in order to avoid any confounding effects of estrogens on the heart [16]. Mice were housed in a temperature and humidity controlled room with alternating 12-hour periods of light and dark and allowed ad-libitum access to water and assigned diet in accordance with Concordia University's animal research ethics committee. Food and water intakes were recorded regularly. In addition, body weight, activity levels were measured throughout the investigation. Fasting and non-fasting glucose measures were taken at the conclusion of the dietary intervention, prior to euthanasia. Fasting glucose measures were obtained following a 12-hour fast. All values were collected with the Precision Xtra glucometer (Abbott Laboratories, Alamaeda, CA). Blood samples for both measures were acquired from a tail bleed and recorded to an accuracy of $0.1 \mathrm{mmol} / \mathrm{L}$.

\subsection{Heart Size}

Wet heart weights were taken in accordance with the standardized procedures set forth by Animal Models of Diabetic Complications Consortium [17]. Immediately following euthanasia by carbon dioxide gas, mice were dissected and blood was extracted from the heart by means of a cardiac puncture. Following removal of blood from the ventricles, hearts were resected and blotted prior to being weighed. Heart weights were measured to an accuracy of 0.1 $\mathrm{mg}$ with the Pioneer balance (OHAUS, Pine Brook, NJ). The left hind leg of the mouse was removed at the level of the femur and stored at -20 degrees Celsius. Mouse legs were then heated to 125 degrees Celsius to allow for easy removal of soft tissues surrounding the tibia. Once tibia had been isolated and given time to dry, length measurements were taken from the mid-point between the medial and lateral condyles to the mid-point between the medial and lateral maleoli. Tibia lengths were measured to an accuracy of 0.01 $\mathrm{mm}$ with a Neiko digital calliper (Montevideo, Uraguay). The heart weight-to-tibia length ratio was computed by dividing the heart weight in milligrams, by the tibia length in millimeters.

\subsection{Glycogen Visualization}

Following the measurement of the wet heart weight, all mouse hearts were imbedded in Optimal Cutting Temperature medium (Fisher Scientific, Pittsburgh, PA), and then slowly cooled in melting isopentane immersed in liquid nitrogen. Eight micrometer sections were cut with a MICROM HM560 cryostar (Thermo Scientific, Layfayette, $\mathrm{CO}$ ). All sections were taken at the level of the papillary muscles and then placed on Super frost slides (Fisher Scientific, Pittsburgh, PA) and allowed to adhere. To visualize intracellular glycogen, sections were stained with a periodic acid solution and Schiff reagent staining kit 395B (Sigma Aldrich, St Louis, MO). Sections were then counterstained with Harris' Hematoxylin to visualize nuclei. Sections were imaged at $200 \mathrm{x}$ magnification with the Olympus BX-60 microscope (Ann Arbour, MI) in established 16-bit colour settings. Colour intensity was determined by setting software to identify all pixels with a given red wavelength associated to the positive staining and presence of glycogen in a set area. Analyses were performed using the ImageJ software (NIH, Besthesda, MD).

Table 1. Detailed Diet Composition

\begin{tabular}{|r|c|c|c|}
\hline & $\begin{array}{c}\text { Agribrands } \\
5075 \text { Charles } \\
\text { River }\end{array}$ & $\begin{array}{c}\text { Harlan } \\
\text { Teklad } \\
\text { TD.110229 }\end{array}$ & $\begin{array}{c}\text { Harlan } \\
\text { Teklad } \\
\text { TD.04524 }\end{array}$ \\
\hline CALORIC CONIUNT & CON & WD & LCHF \\
\hline Carbohydrate (\%) & 63 & 42 & 11 \\
\hline Protein (\%) & 24 & 16 & 46 \\
\hline Fat (\%) & 14 & 42 & 43 \\
\hline Density (kcal/g) & 4.1 & 4.5 & 4.4 \\
\hline Methionine (\%) & 0.39 & 1.3 & 1.3 \\
\hline Cholesterol ( $(\mathbf{k g})$ & 0.01 & 1.5 & 1.5 \\
\hline
\end{tabular}

$\mathrm{CON}=$ Control diet, $\mathrm{WD}=$ high-fat,Western diet, $\mathrm{LCHF}=$ Low-carbohydrate, high-fat diet

\subsection{Statistical Analysis}

All data is presented as mean $\pm \mathrm{SD}$. Analysis was conducted with the SPSS 13.0 statistical package (Chicago, IL). One-way analysis of variance was used to analyze the differences among the groups, and the differences between 2 groups were evaluated by repeated t-tests with a Bonferoni correction. $\mathrm{P}<0.05$ was considered to be statistically significant. 


\section{Results}

A total of 42 mice were used in this investigation (24 YOUNG and 18 MATURE). Of the 24 YOUNG, 9 were assigned to the Control diet (CON), 6 to the Western diet (WD), and 9 to the LCHF diet. Of the 18 MATURE mice, 6 were assigned to the CON diet, 6 to the WD, and 6 to the LCHF diet. Two mice (1 from MATURE WD group and 1 from MATURE LCHF group) were removed from their intervention diet due to dental deformities. Additionally, another 2 mice (1 from MATURE WD group and 1 from MATURE LCHF group) died during the 12-week intervention before completion; cause of death was undeterminable. At baseline, there were no differences in body weight between all groups in both YOUNG and MATURE. At the conclusion of 12 weeks, YOUNG following the LCHF diet weighed less than YOUNG following the WD and CON diets (Table 2). In MATURE, there were no significant differences in weight between all groups at the conclusion of the dietary intervention. In both YOUNG and MATURE, there were no discrepancies in activity level measured in running wheels, fasting glucose, or non-fasting glucose values observed between groups. Data is presented in Table 2.

Table 2. Body weights, activity level and glucose measures for young and mature mice after 12-weeks on diet

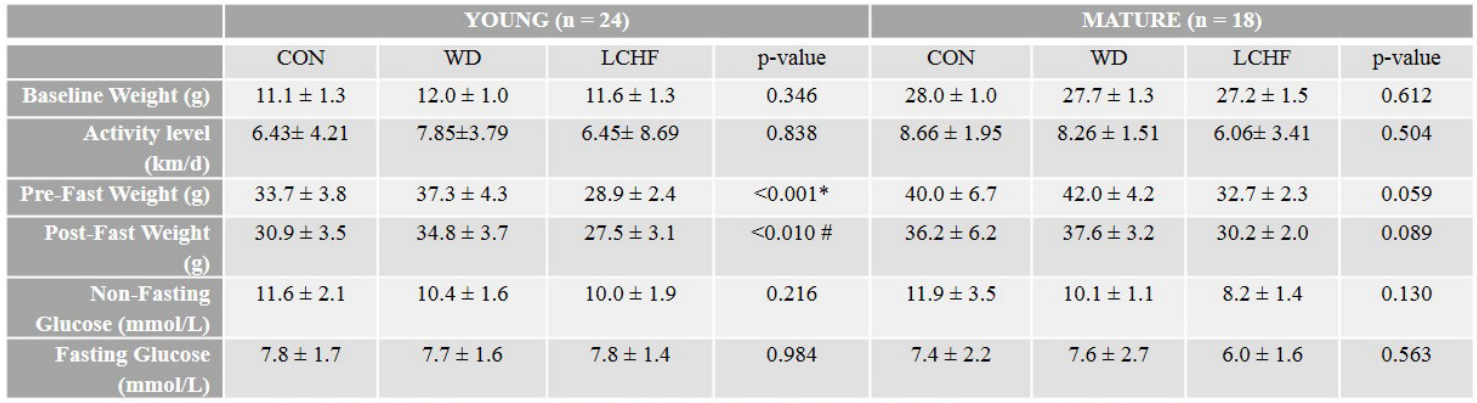

$* \mathrm{P}<0.05$ for $\mathrm{LCHF}$ compared to $\mathrm{CON}$ and $\mathrm{WD} \# \mathrm{P}<0.05$ for LCHF compared to WD only.
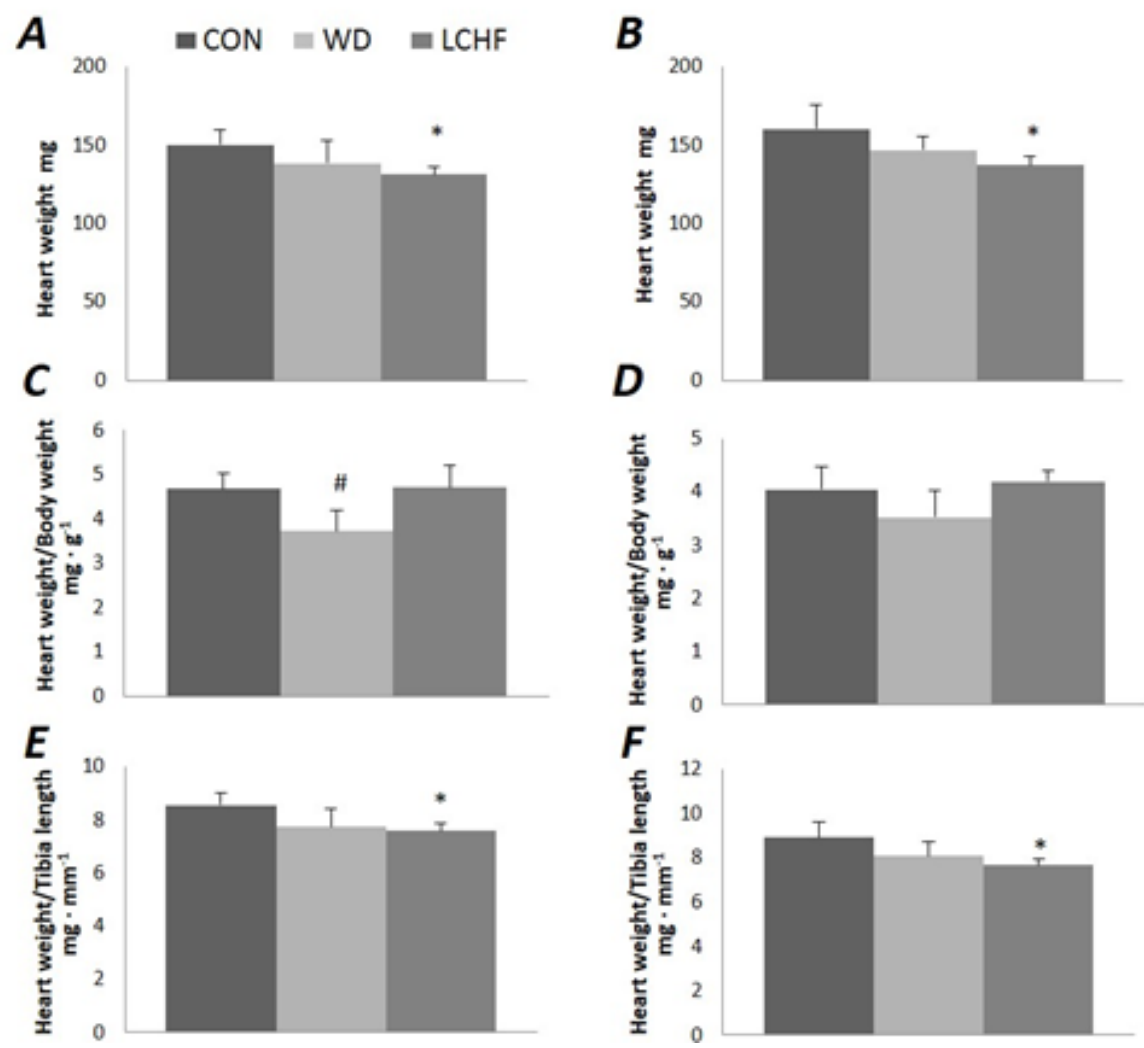

A) Heart weight in YOUNG $(\mathrm{n}=24)$. B) Heart weight in MATURE( $=18)$.C) Heart weight normalized to body weight in YOUNG. D) Heart weight normalized to body weight in MATURE. E) Heart weight normalized to tibia length in YOUNG. F) Heart weight normalized to tibia length in MATURE. ${ }^{*} \mathrm{P}<0.05$ for LCHF compared to CON.\# $\mathrm{P}<0.05$ for WD compared to CON and LCHF

Figure 1. Heart weight in YOUNG and MATURE mice normalized to body weight and tibia length 


\subsection{Heart Size}

After 12-weeks of dietary intervention, there were notable differences between all diet groups (CON, WD and LCHF) in both YOUNG and MATURE. YOUNG LCHF had significantly smaller hearts $(131.2 \pm 4.9 \mathrm{mg})$ than YOUNG CON $(150.1 \pm 9.6 \mathrm{mg})$. There were no notable differences in heart size of YOUNG following the WD $\operatorname{diet}(138.0 \pm 14.4$ $\mathrm{mg}$ ) when compared to both CON and LCHF. Similarly, MATURE following the LCHF diet had significantly smaller hearts $(137.0 \pm 5.5 \mathrm{mg})$ than mice following CON $(160.0 \pm 15.1 \mathrm{mg})$ and WD $(146.5 \pm 8.6 \mathrm{mg})$. As in YOUNG, there was no difference between MATURE CON and WD. When normalized to body weight, differences in heart weight were noted between mice following the WD $(3.73 \pm$ $0.46 \mathrm{mg} / \mathrm{g}$ ) and both groups of mice following the CON diet $(4.67 \pm 0.35 \mathrm{mg} / \mathrm{g})$ and LCHF diet $(4.70 \pm 0.49 \mathrm{mg} / \mathrm{g})$. In MATURE, there were no significant differences noted between CON diet $(4.04 \pm 0.43 \mathrm{mg} / \mathrm{g})$, WD $\operatorname{diet}(3.52 \pm 0.49$ $\mathrm{mg} / \mathrm{g})$, and LCHF diet $(4.19 \pm 0.21 \mathrm{mg} / \mathrm{g})$. Lastly, when normalized to tibia length, YOUNG LCHF exhibited a smaller heart weight-to-tibia length ratio $(7.56 \pm 0.30$ $\mathrm{mg} / \mathrm{mm})$ than YOUNG CON $(8.53 \pm 0.49 \mathrm{mg} / \mathrm{mm})$ with no significant difference between YOUNG WD $(7.72 \pm 0.69$ $\mathrm{mg} / \mathrm{mm}$ ) and YOUNG CON or YOUNG LCHF. A similar pattern occurred in MATURE: MATURE LCHF displayed a smaller heart weight-to-tibia length ratio $(7.66 \pm 0.27$ $\mathrm{mg} / \mathrm{mm})$ when compared to MATURE CON $(8.90 \pm 0.74$ $\mathrm{mg} / \mathrm{mm})$. MATURE WD $(8.07 \pm 0.65 \mathrm{mg} / \mathrm{mm})$ did not differ significantly from either CON or LCHF. Refer to Figure 1 for a summary of the above data.
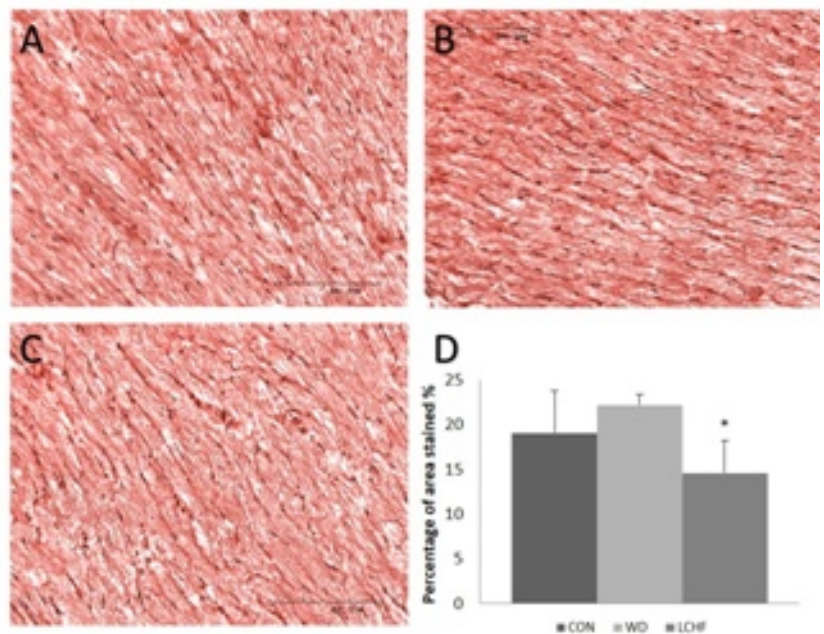

Representative $8 \mu \mathrm{m}$ cardiac tissue sections stained with PAS and Harris' Hematoxylin. 200X magnification. A) Control diet B) Western diet C) Low-carbohydrate, highfat diet. D) Summarized data $(\mathrm{n}=15)^{*} \mathrm{P}<0.05$ for LCHP when compared to Western diet

Figure 2. Cellular area stained positive for glycogen

\subsection{Glycogen Visualization}

The percentage of cardiomyocyte area in YOUNG stained positive for glycogen with the Periodic Acid-Schiff Reagent protocol varied between groups. Significant differences were observed between cardiac sections taken from YOUNG following the LCHF diet $(14.58 \pm 3.60 \%)$ and YOUNG WD $(22.14 \pm 1.22 \%)$. Cardiac sections taken from YOUNG CON (19.05 $\pm 4.69 \%)$ demonstrated a trend towards having an increased percentage of area stained positive for glycogen when compared to LCHF mice, but values did not reach significance $(\mathrm{P} \approx 0.10)$. Data is illustrated in Figure 2.

\section{Discussion}

The objective of the investigation was to determine whether the consumption of a LCHF diet in young subjects affects heart size. If differences in heart size were noted after 12 weeks of dietary intervention, our second objective was to determine whether differences in heart size were due to differences in cross sectional area or disparities in glycogen content. The results of this study suggest that the heart size in both young and mature mice are greatly affected by a LCHF diet. Both young and mature mice following the LCHF diet displayed smaller heart weights than mice following the standard chow Control diet. Although values did not reach significance, there were evident trends that mice given the LCHF diet had smaller heart weights than mice assigned to the high-fat Western diet. This would indicate that differences in heart weight are not solely related to the increased fat content in the diets, but rather a combination of the decreased carbohydrate content and the increased fat content together. With respect to heart weight, responses to the dietary manipulations in mature mice paralleled the responses observed in young mice. This would suggest that age does not have a significant impact on the factors contributing to the decreased heart weights. Our data further suggested that the differences in heart weight observed in young mice may be a result of alterations in glycogen content. The intracellular glycogen content of the young mice assigned to the LCHF diet was decreased in comparison to mice assigned the high-fat Western diet. This data reached significance suggesting that the cardiomyocytes of mice following the LCHF diet retain less glycogen than the cardiomyocytes of mice following the Control diet. This finding demonstrates that differences in heart weight could be related to altered glycogen content. We would expect to see similar trends in glycogen content in the mature mice.

In a recent study published in 2013 [18], similar variations in glycogen content of cardiomyocytes were noted after obese Sprague-Dawley rats were placed on a control diet or LCHF diet for 2 weeks. Previous work in rodents has also suggested that short term use of a LCHF diet can lead to derangements in glycogen stores within the heart [19]. Although the exact mechanisms behind the effects of the LCHF diet on heart size and glycogen stores are unknown, the conserved phosphoinositide 3-kinase (PI3K) pathway has been implicated in the determination of heart size [20]. 
Since insulin signaling is an essential regulator for this pathway, alterations of serum insulin levels in response to a LCHF diet could be responsible for the alterations in heart size observed in our study. The PI3K pathway is also well known to play a key role in glycogen synthase activity [21]. However further investigation into the exact mechanisms behind a decreased heart size in response to a LCHF diet will confirm present hypotheses. With complete picture of the phenomenon, more concrete recommendations can be made to clinicians.

The present study provides important information for clinicians to consider before prescribing a LCHF diet to adolescents or adults. As previously discussed, glycogen availability is extremely important for both developing and stressed hearts. By decreasing cardiac glycogen stores with a LCHF diet, individuals are at risk for not being able to meet metabolic demands during events of cardiac stress, increasing the likelihood of myocardial ischemia. Clinicians prescribing this diet to children should also be cautioned, as prolonged use of a LCHF diet could lead to the development of a smaller heart over the years. One needs to consider all of the risks associated to this diet prior to initiating the intervention. Strengths of the current study include the use of a second control group, the high-fat Western diet group, to control for the high-fat content of the LCHF diet. We were able to distinguish whether the effects of the dietary intervention were due to an increase in the consumption of fats, or the carbohydrate restriction. In addition, our intervention period of 12-weeks allowed for sufficient time for alterations in cardiac remodeling to occur. We noted differences in heart weight that were not apparent after only 2 weeks of dietary intervention [18]. Weaknesses of our investigation include the use of a non-obese murine model. The majority of individuals who are prescribed a LCHF diet are overweight or obese with the goal of weight loss in mind. This may limit the applicability of our findings. Secondly, glycogen content measures were only performed in young mice. Although it is expected that mature mice would demonstrate similar trends as observed in young mice, the added data would strengthen the conclusions.

\section{Conclusions}

The current findings suggest that the use of a LCHF diet can have negative implications for the heart. In this preliminary investigation it was shown that heart size and glycogen content are greatly affected by a LCHF diet in young subjects, with mature subjects demonstrating similar trends. Further investigations will need to examine the potential mechanisms behind this observed response. By understanding the processes involved in the alterations of cardiac glycogen content in response to LCHF diets, more concrete conclusions can be drawn about the overall safety of their use in both children and adults. Clinicians must still remain cautious when prescribing these diets to their patients as long term effects are still unclear. Should LCHF diets impair the heart's ability to perform under stressed conditions, their use in at-risk populations should be questioned and repeated cardiac follow ups during diet adherence are needed.

\section{Acknowledgements}

We are very grateful to the Concordia University Animal Care Facility Manager Aileen Murray and her staff for the housekeeping and caregiving they provided throughout this project. This work was supported by Concordia University.

\section{REFERENCES}

[1] Dietz, W.H. (1998). Health consequences of obesity in youth: childhood predictors of adult disease. Pediatrics, 101(3 Pt 2), 518-525.

[2] de Onis, M., Blössner, M., Borghi, E. (2010). Global prevalence of trends of overweight and obesity among preschool children. The American Journal of Clinical Nutrition, 92(5), 1257-1264.

[3] Atkins, R.C. (1998). Dr. Atkins' New Diet Revolution. Avon Books. New York, NY.

[4] Samaha, F. F., Iqbal, N., Seshadri, P., Chicano, K. L., Daily, D. A., McGrory, J., . . Stern, L. (2003). A low-carbohydrate as compared with a low-fat diet in severe obesity. The New England Journal of Medicine, 348(21), 2074-2081.

[5] Foster, G. D., Wyatt, H. R., Hill, J. O., McGuckin, B. G., Brill, C., Mohammed, B. S., . . Klein, S. (2003). A randomized trial of a low-carbohydrate diet for obesity. The New England Journal of Medicine, 348(21), 2082-2090.

[6] Krebs, N.F., Gao, D., Gralla, J., Collins, J.S., Johnson, S.L. (2010). Efficacy and Safety of a high protein, low carbohydrate diet for weight loss in severely obese adolescents. The Journal Pediatrics, 157, 252-258.

[7] Foo, S. Y., Heller, E. R., Wykrzykowska, J., Sullivan, C. J., Manning-Tobin, J. J., Moore, K. J., . . . Rosenzweig, A. (2009). Vascular effects of a low-carbohydrate highprotein diet. Proceedings of the National Academy of Sciences of the United States of America, 106(36), 15418-15423.

[8] Holloway, C.J., Cochlin, L.E., Emmanuel, Y., Murray, A., Codreanu, I., Edwards, L.M., ... Clarke, K. (2011). A high-fat diet impairs cardiac high-energy phosphate metabolism and cognitive function in healthy human subjects. The American Journal of Clinical Nutrition, 93(4), 748-755.

[9] Wambolt, R.B., Henning, S.L., English, D.R., Dyachkova, Y., Lopaschuk, G.D., Allard, MF. Glucose utilization and glycogen turnover are accelerated in hypertrophied rat hearts during severe low-flow ischemia. The Journal of Molecular and Cellular Cardiology, 31(3),493-502.

[10] Schaefer, S., Ramasamy, R. (1997).Glycogen utilization and ischemic injury in the isolated rat heart. Cardiovascular Research, 35(1),90-98. 
[11] Collins-Nakai, R.L., Noseworthy, D., Lopaschuk, G.D. (1994). Epinephrine increases ATP production in hearts by preferentially increasing glucose metabolism. American Journal of Physiology, 267, H1862-H1871.

[12] Goodwin, G. W., Ahmad, F., Taegtmeyer, H. (1996). Preferential oxidation of glycogen in isolated working rat heart. The Journal of Clinical Investigation, 97, 1409-1416.

[13] Henning, S. L., R. B. Wambolt, B. O. Schonekess, G. D. Lopaschuk, and M. F. Allard. (1996). Contribution of glycogen to aerobic myocardial glucose utilization. Circulation, 93, 1549-1555.

[14] Pederson, B.A., Chen, H., Schroeder, J.M., Shou, W., DePaoli-Roach, A.A., Roach, P.J. (2004). Abnormal Cardiac Development in the Absence of Heart Glycogen. Molecular and Cellular Biology, 24(16), 7179-7187.

[15] Kennedy, A.R., Pissios, P., Out, H., Roberson, R., Xue, B., Asakura, K., ... Maratos-Flier, E. (2007). A high-fat, ketogenic diet induces a unique metabolic state in mice. Endocrinology and Metabolism: American Journal of Physiology, 292(6), E1724-E1739.

[16] Nathan, L., Chaudhuri, G. (1997). Estrogens and atherosclerosis. Annual Reviews of Pharmacology and
Toxicology. 37, 477-515.

[17] AMDCC. (2004). Mouse Morphometry. Animal Models of Diabetic Complications Consortium. AMDCC Protocols, Suppl1, 1-2.

[18] Liu, J., Lloyd, S.G. (2013). High-fat, low-carbohydrate diet alters myocardial oxidative stress and impairs recovery of cardiac function after ischemia and reperfusion in obese rats. Nutrition Research, 33(4), 311-321.

[19] Wang, P., Tate, J.M., Lloyd, S.G. (2008). Low carbohydrate diet decreases myocardial insulin signaling and increases susceptibility to myocardial ischemia. Life Sciences, 83(25-26), 836-44.

[20] Shioi, T., Kang, P.M., Douglas, P.S., Hampe, J., Yballe, C.M., Lawitts, J., Cantley, L.C., Izumo, S. (2000). The conserved phosphoinositide 3-kinase pathway determines heart size in mice. The EMBO Journal, 19(11), 2537-2548.

[21] Moule, S.K., Edgell, N.J., Welsh, G.I., Diggle, T.A., Foulstone, E.J., Heesom, K.J., Proud, C.G., Denton, R.M. (1995). Multiple signalling pathways involved in the stimulation of fatty acid and glycogen synthesis by insulin in rat epididymal fat cells. The Biochemistry Journal, 311(Pt 2), 595-601. 